\section{THU0190 PILOT STUDY OF THE SAFETY AND EFFICACY OF LEFLUNOMIDE (LEF) IN PATIENTS WITH WEGENER'S GRANULOMATOSIS (WG) IN REMISSION}

C Metzler, C Fink, P Lamprecht, WL Gross, E Reinhold-Keller. Clinical Rheumatology, Medical University of Lubeck, Lubeck, Germany

10.1136/annrheumdis-2001.1092

Background WG is a systemic autoimmune disease characterised by small-vessel vasculitis leading to organ damage and the presence of antineutrophil cytoplasmic autoantibodies against proteinase 3 (PR3-ANCA). Treatment of this condition is difficult; the established Fauci?s scheme (daily low dose cyclophosphamide +prednisone) is effective, however it is associated with high morbidity and mortality. As WG is an autoimmune disease, the proven effectiveness of LEF in the treatment of inflammation and autoimmune disease in animal models, and in rheumatoid arthritis (RA), suggests a potential benefit for WG patients.

Objectives To investigate the safety and efficacy of different dose levels of LEF, and to study the maintenance of remission by LEF in 20 WG patients in a 52 week, single-centre, open label study. Patients who completed the study and benefited from LEF were offered continuation of treatment for up to 2 years.

Methods WG patients who have been in complete/incomplete remission induced by Fauci?s scheme received a LEF loading dose (100 mg QD for 3 days) followed by $20 \mathrm{mg}$ QD. After 12 weeks, the dose was increased to $30 \mathrm{mg}$ daily in all patients, following a loading dose (100 mg QD for 2 days). In the absence of complete remission after 24 weeks, the dose was increased to $40 \mathrm{mg}$ daily following a loading dose (100 mg QD for 2 days). The primary efficacy variables measured were organ involvement assessed by the disease extent index (DEI), Birmingham vasculitis activity score (BVAS), the ANCA titer and relapse occurrence. Adverse events (AEs) were observed by the patient as in rheumatoid arthritis clinical trials. All 20 patients who entered the study were included in the efficacy and safety analyses.

Results All patients experienced at least one AE, with the average being 8.3 mentions per patient. The most frequently reported AE was mild upper respiratory infection (40\%). Nine patients (45\%) experienced AEs considered to be causally related to study medication. Hypertension was reported in 3 patients and diarrhoea, nausea, and alopecia were experienced by 2 patients each. One patient who reported a severe AE discontinued from the study due to visual field defects, possibly causally related. The mean total DEI scores were 3.2 at baseline, 2.3 at week 52 and 3.3 at endpoint (defined as the final visit), respectively. The mean total BVAS score was 10.6 at baseline, 5.6 at week 24, 8.4 at week 52 and 9.5 at endpoint. No marked changes over time were observed for either the mean total DEI or BVAS scores. At endpoint, there was no change in ANCA titer in $25 \%$ of patients, however, $55 \%$ showed an increase of more than two titer steps compared to the baseline value. Only one patient showed a decrease from baseline to endpoint. At 24 weeks, 11 patients were increased to $40 \mathrm{mg}$ due to incomplete remission. Nine of these patients experienced additional organ involvement in their disease that necessitated increased corticosteroid doses in 4 patients. One patient discontinued LEF treatment due to lack of efficacy and new renal involvement was considered as a relapse.

Conclusion LEF used for the treatment of WG patients was well tolerated at the doses studied. The extent and activity of WG remained constant throughout the whole study period, and remission was maintained in the majority of patients.

\section{THU0191 FORMAL EDUCATION LEVEL AND CLINICAL STATUS IN RHEUMATOID ARTHRITIS: COMPARISON OF PATIENTS FROM FINLAND AND THE UNITED STATES}

${ }^{1} \mathrm{~T}$ Sokka, ${ }^{2} \mathrm{P}$ Hannonen, ${ }^{1} \mathrm{~T}$ Pincus. 'Division of Rheumatology, Vanderbilt University, Nashville, USA; ${ }^{2}$ Department of Medicine, Jyvaskyla Central Hospital, Jyvaskyla, Finland

\subsection{6/annrheumdis-2001.1093}

Background Formal education level as a marker of socioeconomic status has been found to be associated with clinical status in several cohorts of patients with rheumatoid arthritis (RA) studied in the United States (US). It appeared of interest to analyse a cohort of Finnish patients according to formal education level and compare the results to US patients.

Objectives To analyse 3 cohorts of patients with RA, including 928 patients from Central Finland (Cohort A; mean age 62 years, mean disease duration 11 years), 418 patients from southeastern US (Cohort B; mean age 56 years, mean disease duration 10 years), and 1414 patients from private practices in 8 US cities (Cohort C; mean age 56 years, mean disease duration 12 years). Methods Patients in Cohort A completed a health assessment questionnaire (HAQ), and patients in Cohorth B and C completed its modified version (MHAQ) (range $0-3$ ); all patients completed a visual analogue pain scale (VAS) (range $0-10$ ) and indicated years of formal education. The cut points for high versus low formal education level were 9 years or more (478 patients $-52 \%)$ vs. $<9$ years (450 patients) for Finnish patients in Cohort A; 12 years or more (304 patients $-73 \%$ ) vs. $<12$ years (114 patients) for US patients in Cohort B; and 12 or more years (1049 patients $-74 \%$ ) vs. $<12$ years (365 patients) for US patients in Cohort C. Patients with fewer years of education were older ( $\mathrm{p}<0.001$ in all 3 cohorts) and had longer duration of disease $(\mathrm{p}=0.396-\mathrm{p}<0.001)$. Therefore, all analyses according to education were adjusted for age and duration of disease.

Results In Cohort A, mean HAQ scores were 0.64 in patients with high education versus 0.99 in those with low education ( $p$ $=0.04$ adjusted for age and duration of disease). In Cohort B, mean MHAQ scores were 0.67 in patients with high education versus 1.02 in those with low education $(p<0.001$ adjusted for age and duration of disease). In Cohort C, mean MHAQ scores were 0.58 in patients with high education versus 0.78 in those with low education ( $\mathrm{p}<0.001$ adjusted for age and duration of disease). Pain VAS scores were 2.7, 3.3, and 3.8 in the high education groups vs. 3.9, 5.0, and 4.9 in the low education groups of Cohorts A, B, and C, respectively 9 (all p $<0.001$ adjusted for age and duration of disease).

Conclusion Differences in clinical status according to years of formal education appear similar in Finnish and US patients with RA, although at different education and pain score levels. Years of education may serve as a marker for self-management, and other variables endogenous to the patient, in contrast to variables affected primarily by health professionals, which may importantly affect the course and outcomes of RA and other chronic diseases.

\section{REFERENCE}

1 Callahan LF, Pincus T. Formal education level as a significant marker of clinical status in rheumatoid arthritis. Arthritis Rheum. 1988;31:1346-57 